\title{
ON BROWN-MCCOY RADICAL CLASSES IN CATEGORIES
}

\author{
by S. VELDSMAN
}

(Received 2nd June 1982)

\section{Introduction}

What does a simple ring with unity, a topological $T_{0}$-space and a graph that has at most one loop but may possess edges, have in common? In this note we show that they all are Brown-McCoy semisimple. Suliński has generalised the well-known BrownMcCoy radical class of associative rings (cf. [1]) to a category which satisfies certain conditions. In [3] he defines a simple object, a modular class of objects and the BrownMcCoy radical class as the upper radical class determined by a modular class in a category which, among others, has a zero object and kernels. To include categories like that of topological spaces and graphs, we use the concepts of a trivial object and a fibre. We then follow Suliński and define a simple object, a modular class of objects and then the Brown-McCoy radical class as the upper radical class determined by a modular class.

This definition coincides with Suliński's in his category and yields a non-trivial Brown-McCoy radical class (connectedness) in the category of topological spaces, viz. the class of all indiscrete spaces and in the category of graphs, viz. the class of all graphs $A$ such that if $A$ is non-trivial, then each vertex of $A$ has a loop.

We adopt the notions and notations of [5].

\section{Definitions}

Let $\mathscr{K}$ be any category. For completeness we recall the following notions from [5].

Let $\mathscr{T}$ be the class of objects which satisfies the following conditions:

(T1) If there exists a constant epimorphism $A \rightarrow B$, then $B \in \mathscr{T}$ must hold;

(T2) If there exists a constant monomorphism $C \rightarrow D$, then $C \in \mathscr{T}$ must hold;

(T3) If $T \in \mathscr{T}$, then every morphism $A \rightarrow T$ and $T \rightarrow B$ must be constant.

The elements of $\mathscr{T}$ will be called trivial objects and a subobject $(B, \mu)$ of $A$ is called trivial if $B \in \mathscr{T}$ (or, equivalently, if $\mu$ is constant). Two morphisms $\alpha: A \rightarrow B$ and $\beta: C \rightarrow B$ form a constant pair [4] if, whenever $\delta: D \rightarrow A$ and $\gamma: D \rightarrow C$ are any two morphisms, then the equality $\delta \alpha=\gamma \beta$ must hold. A morphism $\mu: F \rightarrow A$ is called a fibre [4] of $\alpha: A \rightarrow B$ if $\mu \alpha$ is constant and whenever $\delta: C \rightarrow A$ is a morphism such that $\delta \alpha$ and $\mu \alpha$ form a constant pair, then there exists a unique $\delta^{\prime}: C \rightarrow F$ such that $\delta^{\prime} \mu=\delta$. Let $\mathscr{M}$ be the following class of morphisms:

$$
\mathscr{M}=\{\mu \in \text { Mor } \mathscr{K} \mid \mu \text { is a fibre of some morphism in } \mathscr{K}\} .
$$


We suppose Iso $\mathscr{K}$, the class of all $\mathscr{K}$-isomorphisms, is contained in $\mathscr{M}$. Let $\mathscr{E}$ be a class of epimorphisms which is closed under compositions and with Iso $\mathscr{K} \subseteq \mathscr{E}$.

A class of objects $\mathscr{R}$ is a radical class if $\mathscr{R}$ satisfies the following:

$A \in \mathscr{R}$ iff whenever $A \rightarrow B \in \mathscr{E}, B \notin \mathscr{T}$, then there exists a $I \rightarrow B \in \mathscr{M}, I \notin \mathscr{T}$ with $I \in \mathscr{R}$.

A class of objects $\mathscr{S}$ is a semisimple class if $\mathscr{S}$ satisfies the following:

$$
\begin{aligned}
& A \in \mathscr{S} \text { iff whenever } I \rightarrow A \in \mathscr{M}, I \notin \mathscr{T} \text {, then there exists a } \\
& I \rightarrow B \in \mathscr{E}, B \notin \mathscr{T} \text { with } B \in \mathscr{P} .
\end{aligned}
$$

With appropriate choices for $\mathscr{E}$, the above two definitions coincide with the usual Kurosh-Amitsur radical and semisimple classes in algebraic categories, the torsion and torsion-free classes in Abelian categories and the connectednesses and disconnectednesses of topological spaces and graphs. A class of objects $\mathscr{S}$ is called regular if, whenever $A \in \mathscr{S}$ and $I \rightarrow A \in \mathscr{M}, I \notin \mathscr{T}$, then there exists an $I \rightarrow B \in \mathscr{E}, B \notin \mathscr{T}$ with $B \in \mathscr{S}$. Lastly from [5] we need the operators $\mathscr{R}_{\&}$ and $\mathscr{S}_{\mathscr{M}}$ on a class of objects. They are:

$$
\begin{aligned}
& \mathscr{R}_{\mathscr{E}} \mathscr{B}=\{A \in \mathrm{Ob} \mathscr{K} \mid \text { there is no } A \rightarrow B \in \mathscr{E}, B \notin \mathscr{T} \text { with } B \in \mathscr{B}\} \text { and } \\
& \mathscr{S}_{\mathscr{M}} \mathscr{B}=\{A \in \mathrm{Ob} \mathscr{K} \mid \text { there is no } C \rightarrow A \in \mathscr{M}, C \notin \mathscr{T} \text { with } C \in \mathscr{B}\} .
\end{aligned}
$$

If $\mathscr{B}$ is a regular class, then $\mathscr{R}_{\mathscr{B}} \mathscr{B}$ is a radical class and if $\mathscr{C}$ is a radical class, then $\mathscr{S}_{\mathscr{M}} \mathscr{C}$ is a semisimple class.

Definition 2.1. An object $A$ in $\mathscr{K}$ is simple if $A \notin \mathscr{T}$ and whenever $\mu: F \rightarrow A \in \mathscr{M}$, $F \notin \mathscr{T}$, then $(F, \mu)=\left(A, 1_{A}\right)$.

In the categories of rings, groups and modules, the concept of a simple object coincides with the usual concept of a simple ring, simple group and simple module respectively. The category of topological spaces has exactly three non-homeomorphic simple objects, viz. the three two-point spaces. They will be denoted by:

the Sierpińsky space, $D_{C}=(\{0 ; 1\},\{\{0 ; 1\} ;\{0\} ; \phi\})$,

the indiscrete two-point space, $D_{A}=(\{0 ; 1\},\{\{0 ; 1\} ; \phi\})$ and

the discrete two-point space, $D_{D}=(\{0 ; 1\},\{\{0 ; 1\} ;\{0\} ;\{1\} ; \phi\})$.

In the category of undirected graphs (which admits loops) there are exactly six nonisomorphic simple objects, viz. the six two-vertex graphs. They will be denoted by:

$$
\begin{aligned}
& B_{1} \text { with } v\left(B_{1}\right)=\{0 ; 1\} \text { and } e\left(B_{1}\right)=\phi, \\
& B_{2} \text { with } v\left(B_{2}\right)=\{0 ; 1\} \text { and } e\left(B_{2}\right)=\{(0,1)\}, \\
& B_{3} \text { with } v\left(B_{3}\right)=\{0 ; 1\} \text { and } e\left(B_{3}\right)=\{(1,1)\},
\end{aligned}
$$




$$
\begin{aligned}
& B_{4} \text { with } v\left(B_{4}\right)=\{0 ; 1\} \text { and } e\left(B_{4}\right)=\{(0,0) ;(1,1)\}, \\
& B_{5} \text { with } v\left(B_{5}\right)=\{0 ; 1\} \text { and } e\left(B_{5}\right)=\{(0,1) ;(1,1)\} \text { and } \\
& \left.B_{6} \text { with } v\left(B_{6}\right)=\{0 ; 1\} \text { and } e\left(B_{6}\right)=\{0,0) ;(1,1) ;(0,1)\right\} .
\end{aligned}
$$

Let $\mathscr{S}$ be the class of simple objects in $\mathscr{K}$. Then $\mathscr{S}$ is isomorphism closed and if $\mathscr{S}^{\prime} \subseteq \mathscr{S}$, then $\mathscr{S}^{\prime}$ is regular. Hence $\mathscr{R}_{\mathscr{G}} \mathscr{S}^{\prime}$ is a radical class. An interesting, but rather expected property, is that every simple object $A$ must declare itself with respect to any radical class $\mathscr{R}$. I.e. $A \in \mathscr{R}$ or $A \in \mathscr{S}_{\mu} \mathscr{R}$ must hold. Indeed, if $A \notin \mathscr{S}_{\mathcal{M}} \mathscr{R}$, then there is a $\mu: I \rightarrow A \in \mathscr{M}, I \notin \mathscr{T}$ with $I \in \mathscr{R}$. But $A$ simple implies $\mu$ an isomorphism. Hence $A \in \mathscr{R}$ follows.

Definition 2.2. Let $\mathscr{S}$ be a class of simple objects in $\mathscr{K}$. Let $\mu: F \rightarrow A \in \mathscr{M}$. Then:

(1) $\mu$ is an $\mathscr{S}$-maximal fibre of $A$ if there exists an $\alpha: A \rightarrow B \in \mathscr{E}$ with $B \in \mathscr{S}$ such that $\mu$ is a fibre of $\alpha$;

(2) $\mu$ is an $\mathscr{S}$-simple fibre of $A$ if $F \in \mathscr{S}$.

If $\mu: F \rightarrow A$ is an $\mathscr{S}$-maximal fibre of $A$, then $(F, \mu) \neq\left(A, 1_{A}\right)$. Indeed, if $(F, \mu)=\left(A, 1_{A}\right)$ and $\alpha: A \rightarrow B \in \mathscr{E}$ is such that $B \in \mathscr{S}$ and $\mu$ is a fibre of $\alpha$, then $\alpha$ is a constant epimorphism which yields $B \in \mathscr{T}$. This, however, contradicts $B$ a simple object.

Definition 2.3. Let $\mu: F \rightarrow A \in \mathscr{M}$. Then $\mu$ is a retract of $A$ if there exists a $\delta: A \rightarrow F$ such that $\mu \delta=1_{F}$.

Definition 2.4. A class $\mathscr{S}$ of simple objects will be called modular if the following conditions hold:

(M1) If $\delta: P \rightarrow A \in \mathscr{M}$ is an $\mathscr{S}$-simple fibre of $A$, then $\delta$ is a retract of $A$ and there exists an $\mathscr{P}$-maximal fibre $\mu: M \rightarrow A$ of $A$ such that $(P, \delta) \cap(M, \mu)$ exists and is trivial and if $\mu^{\prime}: M^{\prime} \rightarrow A$ is any other $\mathscr{S}$-maximal fibre of $A$ such that $(P, \delta) \cap\left(M^{\prime}, \mu^{\prime}\right)$ $=(P, \delta) \cap(M, \mu)$, then $(M, \mu) \leqq\left(M^{\prime}, \mu^{\prime}\right)$ must hold.

(M2) If $\delta: P \rightarrow A \in \mathscr{M}$ and $\sigma: L \rightarrow P$ is an $\mathscr{P}$-maximal fibre of $P$, then $\sigma \delta \in \mathscr{M}$ must hold.

Obviously, in categories where $\mathscr{M}$ is closed under composition, (M2) is trivially satisfied. Every modular class is regular. Hence, if $\mathscr{S}$ is a modular class, then $\mathscr{R}_{\mathscr{G}} \mathscr{S}$ is a radical class and will be called the Brown-McCoy radical class determined by $\mathscr{S}$.

\section{Examples}

3.1. If $\mathscr{K}$ satisfies the conditions of Suliński [3], then the above definition of a modular class coincides with his if $\mathscr{E}$ is the class of all normal epimorphisms. Indeed, $(M, \mu)$ and $\left(M^{\prime}, \mu^{\prime}\right)$ are maximal ideals and if $(M, \mu) \leqq\left(M^{\prime}, \mu^{\prime}\right)$ then $(M, \mu)=\left(M^{\prime}, \mu^{\prime}\right)$. Because the only trivial ideal is of the form $(0, \omega)$, it follows that $(M, \mu)$ is unique with respect to the property $(P, \delta) \cap(M, \mu)=(0, \omega)$. For the categories of associative rings and alternative rings, Suliński has shown that any abstract class of simple rings with unity is 
modular. The ring theoretic Brown-McCoy radical is the smallest radical class among them, i.e. the upper radical determined by the class of all simple rings with unity.

3.2. Let $\mathscr{K}$ be the category of all topological spaces and continuous functions with $\mathscr{E}$ the class of all onto continuous functions. We show that, in this case, there is only one non-trivial Brown-McCoy radical class (connectedness), viz. $\mathscr{R}_{\diamond}\left\{D_{c}\right\}=\{$ all indiscrete spaces $\}$. Let $\mathscr{S}=\left\{D_{C}\right\}$. Obviously $\mathscr{S}$ satisfies (M2) and to see (M1), suppose $D_{C}$ is a subspace of a space $X$ with $j: D_{C} \rightarrow X$ the inclusion. Because $\{0\}$ is open in $D_{C}$, there exists an open subset $V$ in $X$ such that $V \cap\{0 ; 1\}=\{0\}$. Let $U$ be the union of all the open subsets $V$ of $X$ such that $V \cap\{0 ; 1)=\{0\}$. Then $U$ is open in $X$. Define $f: X \rightarrow D_{C}$ by $f(U)=\{0\}$ and $f(X-U)=\{1\}$. Then $f$ is an onto continuous function, if $=1_{D_{C}}$ and $D_{C} \cap f^{-1}(1)=D_{C} \cap(X-U)=\{1\}$. If $g: X \rightarrow D_{C}$ is any other onto continuous function such that $D_{C} \cap g^{-1}(x)=\{1\}$ for some $x \in\{0 ; 1\}$, then $f^{-1}(1) \subseteq g^{-1}(x)$. Indeed, if $x=0$, then $\{1\}$ $=D_{C} \cap g^{-1}(0)$ must be open in $D_{C}$ which is not possible. Hence $x=1$ and if $W=g^{-1}(0)$, then $W$ is open in $X$ and $W \subseteq U$. Thus $f^{-1}(1)=X-U \subseteq X-W=g^{-1}(1)$ follows. Hence

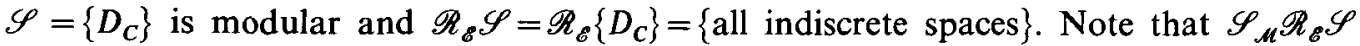
$=\left\{\right.$ all $T_{0}$-spaces $\}$. If $\mathscr{S}=\left\{D_{A}\right\}$ it can also be shown that $\mathscr{S}$ is modular, but $\mathscr{R}_{\mathscr{B}} \mathscr{S}$ is the class of all trivial spaces and $\mathscr{S}_{\mathscr{M}} \mathscr{R}_{\mathscr{S}} \mathscr{S}$ is the class of all topological spaces. In fact, if $\mathscr{B}$ is any regular class of topological spaces such that $D_{A} \in \mathscr{B}$, then $\mathscr{R}_{\mathscr{B}} \mathscr{B}$ is the class of all trivial spaces. If $\mathscr{S}=\left\{D_{D}\right\}$, then $\mathscr{S}$ is not modular. To this end we need the following lemma of which the proof is elementary:

Lemma. Let $D_{D}$ be a subspace of $X$. Then $D_{D}$ is a retract of $X$ iff there exists an open and closed subset $U$ of $X$ such that $0 \in U$ and $1 \in X-U$.

Let $Z=\{0 ; 1 ; 2\}$ with $\{0 ; 2\},\{1 ; 2\}$ and $\{2\}$ open in $Z$. Then $D_{D}$ is a subspace of $Z$, but $Z$ has no open and closed subset $U$ with $0 \in U$ and $1 \in X-U$. Hence $D_{D}$ is not a retract of $Z$ and $\mathscr{S}=\left\{D_{D}\right\}$ is not modular. Thus the only modular class which yields a nontrivial Brown-McCoy radical class is the class $\mathscr{S}=\left\{D_{D}\right\}$.

3.3. Let $\mathscr{K}$ be the category of all undirected graphs (which admit loops) and graph homomorphisms. Let $\mathscr{E}$ be the class of all onto homomorphisms. We show that, in this case, there is only one non-trivial Brown-McCoy radical class, viz. $\mathscr{R}_{\mathscr{B}}\left\{B_{5}\right\}=\{A \mid$ if $A$ is non-trivial, then each vertex of $A$ has a loop $\}$. Let $\mathscr{S}=\left\{B_{5}\right\}$. (M2) follows trivially and to show that $\mathscr{S}$ is modular, we show the validity of (M1). Suppose $B_{5}$ is a subgraph of $A$. Let $X$ be the biggest subgraph of $A$ such that $0 \in v(X)$ and $e(X)=\phi$. There is at least one such subgraph, viz. the loopless vertex 0 . Define $\theta: A \rightarrow B_{5}$ by $\theta(v(X))=\{0\}$ and $\theta(v(A)-v(X))=\{1\}$. Then $\theta$ is an onto homomorphism and if $j: B_{5} \rightarrow A$ is the inclusion, then $j \theta=1_{B_{5}}$. Furthermore, $\theta^{-1}(1) \cap B_{5}=(A-X) \cap B_{5}$ which is the vertex 1 with a loop. If $\alpha: A \rightarrow B_{5}$ is any other onto homomorphism such that $\alpha^{-1}(x) \cap B_{5}=\theta^{-1}(1) \cap B_{5}$ for some $x \in v\left(B_{5}\right)$, then $x=1$ and $\theta^{-1}(1) \subseteq \alpha^{-1}(1)$ follows by remembering that $(0,0) \notin e\left(B_{5}\right)$ and using the definition of the subgraph $X$. Hence $\mathscr{S}=\left\{B_{5}\right\}$ is modular, $\mathscr{R}_{\mathscr{S}} \mathscr{S}=\mathscr{R}_{\mathscr{S}}\left\{B_{5}\right\}$ $=\{A \mid$ if $A$ is non-trivial, then each vertex has a loop $\}$ and

$\mathscr{S}_{\mathscr{M}} \mathscr{R}_{\mathscr{S}}\left\{B_{5}\right\}=\{A \mid A$ has at most one loop but may possess edges $\}$. 
If $\mathscr{S}=\left\{B_{6}\right\}$ it can be shown that $\mathscr{S}$ is modular. However, $\mathscr{R}_{8}\left\{B_{6}\right\}$ is the class of all trivial graphs. In fact, if $\mathscr{C}$ is any regular class of graphs such that $B_{6} \in \mathscr{C}$, then $\mathscr{R}_{8} \mathscr{B}$ is the class of all trivial graphs. Each of the graphs $B_{1}, B_{2}, B_{3}$ and $B_{4}$ can be subgraphs of a graph without being a retract. Hence any class of simple graphs which contains one of $B_{1}, B_{2}, B_{3}$ or $B_{4}$ is not modular.

A final remark concerns the hereditariness of the Brown-McCoy radical class. Rashid and Wiegandt [2] have shown that in a category with, among others, a zero object and kernels, every radical class $\mathscr{R}_{\mathscr{G}} \mathscr{S}$ determined by a modular class $\mathscr{S}$ (where $\mathscr{E}$ is the class of all normal epimorphisms) is hereditary. Although, in this general setting, I have not been able to prove this result, the Brown-McCoy connectednesses in Top and Graph behave nicely with respect to this property, i.e., they both are hereditary. In fact, they are the only hereditary connectednesses in these two categories (cf. [6]).

\section{REFERENCES}

1. N. J. Divinsky, Rings and Radicals (George Allen and Unwin Ltd., London, 1975).

2. M. A. Rashid and R. Wiegand, The hereditariness of the upper radical, Acta Math. Sci. Hung. 24 (1973), 343-347.

3. A. Sulinski, The Brown-McCoy radical in categories, Fund. Math. 59 (1966), 23-41.

4. J. A. Tiller, Component subcategories, Quaestiones Mathematicae 4 (1980), 19-40.

5. S. Veldsman, On the characterization of radical and semisimple classes in categories, Comm. in Algebra 10 (1982), 913-938.

6. R. WIEGANDT, A condition in general radical theory and its meaning for rings, topological spaces and graphs, Acta Math. Acad. Sci. Hung. 26 (1975), 233-240.

Department of Mathematics

Rand Afrikaans University

P.O. Box 524

JOHANNESBURG 2000

South Africa 